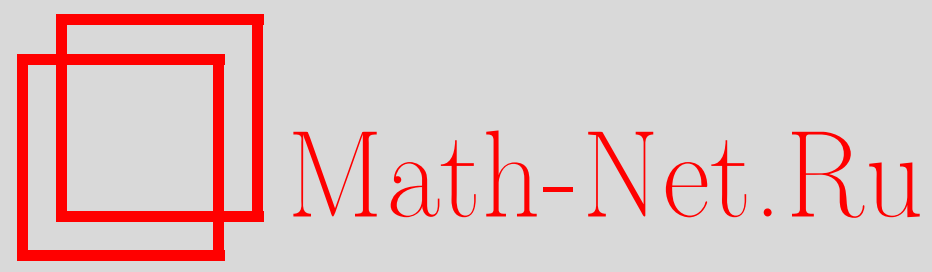

А. А. Карацуба, Дробные моменты и нули $\zeta(s)$ на критической прямой, Матем. заметки, 2002, том 72, выпуск 4, 502508

DOI: https://doi.org/10.4213/mzm439

Использование Общероссийского математического портала Math-Net.Ru подразумевает, что вы прочитали и согласны с пользовательским соглашением http://www.mathnet.ru/rus/agreement

Параметры загрузки:

IP : 54.198 .67 .100

26 апреля 2023 г., 18:01:00

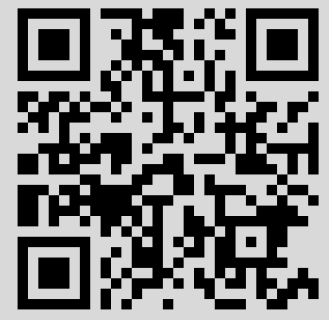




\section{ДРОБНЫЕ МОМЕНТЫ И НУЛИ $\zeta(s)$ НА КРИТИЧЕСКОЙ ПРЯМОЙ}

\section{А. А. Карацуба}

Установлена связь между дробными моментами дзета-функции Римана и количеством ее нулей, лежащих на критической прямой.

Библиографория: 10 названий.

Пусть $N_{0}(T)$ - количество нулей $\zeta(1 / 2+i t)$ на промежутке $0<t \leqslant T$. Здесь и ниже $\zeta(s)$ - дзета-функция Римана, $s=\sigma+i t, i^{2}=-1, \sigma, t$-вешественные числа, $T \geqslant T_{1}>0$, прямая $\operatorname{Re} s=1 / 2$ на $s$-плоскости назьвается критической прямой.

Харди и Литтлвуд [1] доказали, что справедливо следующее неравенство:

$$
N_{0}(T) \geqslant c T
$$

где $c>0$ - абсолютная постоянная.

Сельберг [2] получил правильный порядок нижней оценки для $N_{0}(T)$ :

$$
N_{0}(T) \geqslant c T \log T
$$

При доказательстве [2] Сельберг пользуется оригинальной идеей умножения $\zeta(s)$ на “успокаивающую” функцию $\varphi(s) \varphi(1-s)$ такую, что множество нечетных нулей новой функции $F(s)=\zeta(s) \varphi(s) \varphi(1-s)$ совпадает с множеством нечетных нулей $\zeta(s)$ на рассматриваемом промежутке, а сама $F(s)$ "в среднем" близка к константе. Это позволяет “погасить" неточности применяемьх неравенств и получить (2).

Цель настоящей статьи - показать, что знание правильного порядка дробного момента $\zeta(s)$ на прямой $\operatorname{Re} s=1 / 2$ дает возможность получить результат более точный, чем (1) (правда, менее точный, чем (2)), теми средствами, которыми пользовались Харди и Литтлвуд.

Для доказательства теоремы понадобятся две леммы: одна - о дробных моментах $|\zeta(1 / 2+i t)|$, другая - о приближении функции Римана на $Z(t)$ начальным отрезком ряда Дирихле.

ЛЕмма 1. При любом натуральном числе $m$ справедливы следующие неравенcmsa:

$$
T(\log T)^{1 / m^{2}} \ll \int_{0}^{T}\left|\zeta\left(\frac{1}{2}+i t\right)\right|^{2 / m} d t \ll T(\log T)^{1 / m^{2}} .
$$


ДокАЗАТЕльство этой леммы см., например, в [3], где упомянуты, кроме работ Баласубраманиана и Рамачандры, статья Хиз-Брауна [4] и статья Ютилы [5], в которой доказано, что постоянные в знаках « леммы 1 абсолютные.

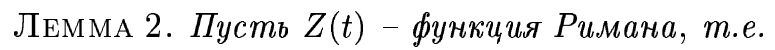

$$
Z(t)=e^{i \theta(t)} \zeta\left(\frac{1}{2}+i t\right), \quad e^{i \theta(t)}=\pi^{-i t / 2} \frac{\Gamma(1 / 4+i t / 2)}{|\Gamma(1 / 4+i t / 2)|} .
$$

Тогда функиия $Z(t)$ при вещественных $t$ принимает вещественные значения, ее вещественные нули являются нулями $\zeta(s)$, лежсащими на критической прямой, и при $t \geqslant 2 \pi$ справедлива следующая формула:

$$
Z(t)=2 \sum_{n \leqslant \sqrt{t /(2 \pi)}} \frac{1}{\sqrt{n}} \cos \left(t \log \sqrt{\frac{t}{2 \pi}}-t \log n-\frac{t}{2}-\frac{\pi}{8}\right)+O\left(t^{-1 / 4} \log t\right),
$$

причем постоянная в знаке $О$ абсолютная.

ДокАЗАТЕЛьСТво этой леммы см., например, в [6].

Упростим правую часть (3), предполагая, что $t$ меняется на коротком промежутке. Пусть $T \leqslant t \leqslant T+H, H \leqslant T \log ^{-1} T$. Рассмотрим две функции $\theta_{1}(t)$ и $\theta_{2}(t)$ :

$$
\theta_{1}(t)=t \log \sqrt{\frac{t}{2 \pi}}-\frac{t}{2}-\frac{\pi}{8}, \quad \theta_{2}(t)=t \log \sqrt{\frac{T}{2 \pi}}-\frac{T}{2}-\frac{\pi}{8}+\frac{(t-T)^{2}}{4 t} .
$$

Пользуясь формулой Тейлора и теоремой Лагранжа о конечных приращениях, последовательно находим

$$
\begin{array}{r}
\theta_{1}(t)-\theta_{2}(t)=t \log \sqrt{\frac{t}{T}}-\frac{t-T}{2}-\frac{(t-T)^{2}}{4 t} \\
=-\frac{1}{2} t \log \left(1-\frac{t-T}{t}\right)-\frac{t-T}{2}-\frac{(t-T)^{2}}{4 t}=O\left(H^{3} T^{-2}\right) \\
\cos \left(\theta_{1}(t)-t \log n\right)-\cos \left(\theta_{2}(t)-t \log n\right)=O\left(H^{3} T^{-2}\right) \\
Z(t)=2 \sum_{n \leqslant P} \frac{1}{\sqrt{n}} \cos \left(t \log \frac{P}{n}+\varphi(t)\right)+O\left(H^{3} T^{-7 / 4}\right)+O\left(T^{-1 / 4} \log T\right)
\end{array}
$$

где

$$
P=\sqrt{\frac{T}{2 \pi}}, \quad \varphi(t)=-\frac{T}{2}-\frac{\pi}{8}+\frac{(t-T)^{2}}{4 t} .
$$

Если, кроме того, $0 \leqslant u \leqslant 1$, то

$$
\begin{gathered}
\varphi(t+u)-\varphi(t)=O\left(H T^{-1}\right) \\
Z(t+u)=2 \sum_{n \leqslant P} \frac{1}{\sqrt{n}} \cos \left((t+u) \log \frac{P}{n}+\varphi(t)\right) \\
+O\left(H^{3} T^{-7 / 4}\right)+O\left(T^{-1 / 4} \log T\right)+O\left(H T^{-3 / 4}\right) .
\end{gathered}
$$

Последней формулой для $Z(t+u)$ мы и будем пользоваться ниже. 
Tеорема. Cуществует постоянная $c>0$ такая, что при $T \geqslant T_{1}>0$ выполняется неравенство

$$
N_{0}(T) \geqslant c T \log ^{1 / 8} T \text {. }
$$

ДокАЗАТЕльСтво. 1. Пусть $T \geqslant T_{1}>0, \log \log T \leqslant h^{-1}=h^{-1}(T) \leqslant \log T$. Более точно значение $h=h(T)$ определим позднее. Рассмотрим два интеграла $j_{1}(t)$ и $j_{2}(t)$ :

$$
j_{1}(t)=\int_{0}^{h}|Z(t+u)| d u, \quad j_{2}(t)=\left|\int_{0}^{h} Z(t+u) d u\right| .
$$

Определим множество $E$ чисел $t, 0<t \leqslant T$, следуюшим образом: $t \in E$, если вьполняется неравенство

$$
j_{1}(t)>j_{2}(t) .
$$

Заметим, что если $t \in E$, то функция $Z(t+u)$ на промежутке $0<u<h$ меняет знак, т.е. на этом промежутке $Z(t+u)$ имеет нуль нечетного порядка.

Пользуясь определением $E$, последовательно получаем

$$
\begin{aligned}
\sqrt{j_{1}(t)} & \geqslant \sqrt{j_{1}(t)}-\sqrt{j_{2}(t)}, \\
\int_{E} \sqrt{j_{1}(t)} d t & \geqslant \int_{E}\left(\sqrt{j_{1}(t)}-\sqrt{j_{2}(t)}\right) d t=\int_{0}^{T}\left(\sqrt{j_{1}(t)}-\sqrt{j_{2}(t)}\right) d t \\
& =\int_{0}^{T} \sqrt{j_{1}(t)} d t-\int_{0}^{T} \sqrt{j_{2}(t)} d t .
\end{aligned}
$$

Применяя к интегралу левой части последнего соотношения неравенство Коши, приходим к основному неравенству:

$$
I_{1} \leqslant I_{2}+\sqrt{\mu(E) I_{3}}
$$

где

$$
I_{1}=\int_{0}^{T} \sqrt{j_{1}(t)} d t, \quad I_{2}=\int_{0}^{T} \sqrt{j_{2}(t)} d t, \quad I_{3}=\int_{0}^{T} j_{1}(t) d t
$$

$\mu(E)$ - мера множества $E$.

2. Оценим $I_{1}$ снизу. Прежде всего, имеем следующие неравенства:

$$
\begin{gathered}
\left(\int_{0}^{h} \sqrt{|Z(t+u)|} d u\right)^{2} \leqslant h \int_{0}^{h}|Z(t+u)| d u, \\
\int_{0}^{h} \sqrt{|Z(t+u)|} d u \leqslant \sqrt{h} \sqrt{j_{1}(t)} .
\end{gathered}
$$

Отсюда и из определения $I_{1}$ приходим к такой оценке:

$$
\begin{aligned}
I_{1} & =\int_{0}^{T} \sqrt{j_{1}(t)} d t \geqslant h^{-1 / 2} \int_{0}^{T} \int_{0}^{h}|Z(t+u)|^{1 / 2} d u d t \\
& \geqslant \sqrt{h} \int_{h}^{T}|Z(t)|^{1 / 2} d t \geqslant \sqrt{h} \int_{1}^{T}\left|\zeta\left(\frac{1}{2}+i t\right)\right|^{1 / 2} d t .
\end{aligned}
$$


Наконец, пользуясь леммой 1 при $m=4$, получаем нужную нам оценку $I_{1}$ снизу:

$$
I_{1} \gg \sqrt{h} T \log ^{1 / 16} T \text {. }
$$

3. Оценим $I_{3}$ сверху. Пользуясь определением $I_{3}$ и в нужном месте леммой 1 при $m=2$, последовательно получаем

$$
I_{3}=\int_{0}^{T} \int_{0}^{h}|Z(t+u)| d u d t \leqslant h \int_{0}^{T+1}\left|\zeta\left(\frac{1}{2}+i t\right)\right| d t \ll h T \log ^{1 / 4} T .
$$

4. Оценим $I_{2}$ сверху. Возводя $I_{2}$ в четвертую степень и применяя неравенство Гёльдера, получаем

$$
I_{2}^{4} \leqslant T^{3} \int_{0}^{T} j_{2}^{2}(t) d t=T^{3}\left(A_{1}+A_{2}\right)
$$

где

$$
A_{1}=\int_{0}^{T L_{1}} j_{2}^{2}(t) d t, \quad A_{2}=\int_{T L_{1}}^{T} j_{2}^{2}(t) d t, \quad L_{1}=\log ^{-1} T
$$

Так как

$$
j_{2}^{2}(t)=\left|\int_{0}^{h} Z(t+u) d u\right|^{2} \leqslant h \int_{0}^{h}|Z(t+u)|^{2} d u
$$

то

$$
A_{1} \ll h^{2} \int_{0}^{T L_{1}}\left|\zeta\left(\frac{1}{2}+i t\right)\right|^{2} d t \ll h^{2} T \ll h T .
$$

Возьмем $H=T^{7 / 12} L_{1}, \nu_{1}=\left[T^{5 / 12}\right], \nu_{2}=\left[T^{5 / 12} L\right], L=\log T$. Очевидно следующее неравенство:

$$
A_{2} \leqslant \sum_{\nu=\nu_{1}}^{\nu_{2}} B_{\nu},
$$

где

$$
B_{\nu}=\int_{H \nu}^{H \nu+H} j_{2}^{2}(t) d t
$$

Будем оценивать сверху $B_{\nu}$. Полагая $T_{1}=H \nu, T_{1} \leqslant t \leqslant T_{1}+H, P_{1}=\sqrt{T_{1} /(2 \pi)}$, по формуле (4) находим

$$
Z(t+u)=2 \sum_{n \leqslant P_{1}} \frac{1}{\sqrt{n}} \cos \left((t+u) \log \frac{P_{1}}{n}+\varphi(t)\right)+O\left(L^{-9}\right)
$$

где

$$
\varphi(t)=-\frac{T_{1}}{2}-\frac{\pi}{8}+\frac{\left(t-T_{1}\right)^{2}}{4 t} .
$$


Преобразуем сначала $j_{2}(t)$. Разбивая промежуток суммирования по $n$ в $(12)$ на два: $n \leqslant P_{2}$, где $P_{2}=P_{1} e^{-1 / h}$, и $P_{2}<n \leqslant P_{1}$, получаем

$$
\begin{aligned}
j_{2}(t)= & \left|\int_{0}^{h} Z(t+u) d u\right| \\
\ll & \left|\int_{0}^{h} \sum_{n \leqslant P_{2}} \frac{1}{\sqrt{n}} \cos \left((t+u) \log \frac{P_{1}}{n}+\varphi(t)\right) d u\right| \\
& +\int_{0}^{h}\left|\sum_{P_{2}<n \leqslant P_{1}} \frac{1}{\sqrt{n}} \cos \left((t+u) \log \frac{P_{1}}{n}+\varphi(t)\right)\right| d u+h L_{1}^{3} .
\end{aligned}
$$

В последних формулах запишем косинус в виде суммы двух экспонент. Проводя интегрирование в первом интеграле, а затем переходя к неравенствам, приходим к следующему соотношению:

$$
\begin{aligned}
j_{2}(t) \ll & \left|\sum_{n \leqslant P_{2}} \frac{1}{\sqrt{n} \log \left(P_{1} / n\right)} n^{i(t+h)}\right|+\left|\sum_{n \leqslant P_{2}} \frac{1}{\sqrt{n} \log \left(P_{1} / n\right)} n^{i t}\right| \\
& +\int_{0}^{h}\left|\sum_{P_{2}<n \leqslant P_{1}} \frac{1}{\sqrt{n}} n^{i(t+u)}\right| d u+h L_{1}^{3} .
\end{aligned}
$$

Возведем обе части последнего неравенства в квадрат и применим неравенство Коши. Вспоминая определение $B_{\nu}$, находим

$$
\begin{gathered}
B_{\nu} \ll D_{1}+D_{2}+D_{3}+D_{4}, \\
D_{1}=D_{1}(h)=\int_{T_{1}}^{T_{1}+H}\left|\sum_{n \leqslant P_{2}} \frac{1}{\sqrt{n} \log \left(P_{1} / n\right)} n^{i(t+h)}\right|^{2} d t, \quad D_{2}=D_{1}(0), \\
D_{3}=h^{2} \int_{T_{1}}^{T_{1}+H}\left|\sum_{P_{2}<n \leqslant P_{1}} \frac{1}{\sqrt{n}} n^{i\left(t+u_{1}\right)}\right|^{2} d t, \quad 0 \leqslant u_{1} \leqslant h, \quad D_{4}=h^{2} H L_{1}^{6} \ll h H .
\end{gathered}
$$

Так как $H$ больше корня квадратного из $T_{1}$, то интегралы $D_{1}, D_{2}, D_{3}$ оцениваются "диагональю". Оценим, например, $D_{1}$. Следующие преобразования очевидны:

$$
\begin{aligned}
D_{1} & \ll \int_{-\infty}^{+\infty} e^{-(t / H)^{2}}\left|\sum_{n \leqslant P_{2}} \frac{1}{\sqrt{n} \log \left(P_{1} / n\right)} n^{i\left(T_{1}+h+t\right)}\right|^{2} d t \\
= & \sum_{n, m \leqslant P_{2}} \frac{1}{\sqrt{n m} \log \left(P_{1} / n\right) \log \left(P_{1} / m\right)}\left(\frac{n}{m}\right)^{i\left(T_{1}+h\right)} \int_{-\infty}^{+\infty} e^{-(t / H)^{2}+i t \log (n / m)} d t \\
= & \sqrt{\pi} H \sum_{n, m \leqslant P_{2}} \frac{1}{\sqrt{n m} \log \left(P_{1} / n\right) \log \left(P_{1} / m\right)}\left(\frac{n}{m}\right)^{i\left(T_{1}+h\right)} e^{-((H / 2) \log (n / m))^{2}} \\
& \ll H \sum_{n \leqslant P_{2}} \frac{1}{n \log ^{2}\left(P_{1} / n\right)} e^{-((H / 2) \log (n / m))^{2} .} \\
& +H \sum_{m \neq n \leqslant P_{2}} \frac{1}{\sqrt{n m} \log \left(P_{1} / n\right) \log \left(P_{1} / m\right)} e^{-}
\end{aligned}
$$


Так как функция $x \log ^{2}\left(P_{1} / x\right)$ при $1 \leqslant x \leqslant P_{2}$ монотонно возрастает, то первая сумма оценивается сверху интегралом, т.е.

$$
\sum_{n \leqslant P_{2}} \frac{1}{n \log ^{2}\left(P_{1} / n\right)} \leqslant \frac{1}{\log ^{2} P_{1}}+\int_{1}^{P_{2}} \frac{d x}{x \log ^{2}\left(P_{1} / x\right)} \ll \log ^{-1} \frac{P_{1}}{P_{2}} \ll h .
$$

Далее, при $n \neq m$ очевидны неравенства $(n>m)$ :

$$
\begin{gathered}
\left|\log \frac{n}{m}\right|=\left|\log \left(1+\frac{n-m}{m}\right)\right| \geqslant \log \left(1+\frac{1}{m}\right) \geqslant \log \left(1+\frac{1}{P_{2}}\right) \geqslant \frac{1}{2 P_{2}} \geqslant \frac{1}{\sqrt{T_{1}}}, \\
H \sum_{n \neq m \leqslant P_{2}} \frac{1}{\sqrt{n m} \log \left(P_{1} / n\right) \log \left(P_{1} / m\right)} e^{-((H / 2) \log (n / m))^{2}} \ll 1 .
\end{gathered}
$$

Тем самьм, для $D_{1}, D_{2}$ получили оценки

$$
D_{1} \ll H h, \quad D_{2} \ll H h .
$$

Точно так же оценивается $D_{3}$ :

$$
D_{3} \ll h^{2} H \sum_{P_{2}<n \leqslant P_{1}} \frac{1}{n} \ll h^{2} H \log \frac{P_{1}}{P_{2}} \ll h H .
$$

Таким образом, из оценок $D_{j}, 1 \leqslant j \leqslant 4$, и формул $(13),(10),(9),(8)$ последовательно получаем

$$
B_{\nu} \ll h H, \quad A_{2} \ll h T, \quad I_{2}^{4} \ll h T^{4}, \quad I_{2} \ll h^{1 / 4} T .
$$

Тем самьм, мы пришли к нужной оценке $I_{2}$.

5. Оценим снизу $\mu(E)$. Для этого подставим найденные оценки для $I_{1}, I_{2}, I_{3}$ в $(5)$. Вводя абсолютные положительные постоянные $c_{1}, c_{2}, c_{3}$, которые содержатся в знаках « соответствуюших оценок для $I_{j}, j=1,2,3$, получаем

$$
\begin{gathered}
c_{1} \sqrt{h} T \log ^{1 / 16} T-c_{2} h^{1 / 4} T \leqslant \sqrt{c_{3} \mu(E) h T \log ^{1 / 4} T} \\
\sqrt{c_{3} \mu(E) T \log ^{1 / 4} T} \geqslant T\left(c_{1} \log ^{1 / 4} T-c_{2} h^{-1 / 4}\right) .
\end{gathered}
$$

Возьмем теперь $h=h(T)$ таким:

$$
h=\left(\frac{2 c_{2}}{c_{1}}\right)^{4} \log ^{-1 / 4} T .
$$

Тогда будем иметь неравенство

$$
c_{1} \log ^{1 / 16} T-c_{2} h^{-1 / 4} \geqslant \frac{1}{2} c_{1} \log ^{1 / 16} T
$$


и, следовательно,

$$
\mu(E) \geqslant \frac{1}{c_{3}}\left(\frac{c_{1}}{2}\right)^{2} T \log ^{-1 / 8} T .
$$

Отсюда и из (14) уже следует утверждение теоремы. Действительно, разобьем промежуток $(0, T)$ на промежутки вида $(h \nu, h \nu+h), \nu=0,1, \ldots, \nu_{3}=\left[T h^{-1}\right]$. По крайней мере $K$ из этих промежутков, где

$$
K=\left[\left(\frac{1}{c_{3}}\left(\frac{c_{1}}{2}\right)^{2} T \log ^{-1 / 8} T\right) h^{-1}\right]
$$

содержат хотя бы одну точку $t$ из $E$. Если бы это было не так, то для $\mu(E)$ получилась бы верхняя гранища, которая меньше нижней. Но точки $t \in E$ характеризуются тем, что на промежутке $(t, t+h)$ функция $Z(u)$ меняет знак, т.е. на этом промежутке сушествует нуль нечетного порядка $Z(u)$. Следовательно, количество нулей $Z(t)$ на $(0, T)$ будет не меньше, чем

$$
\frac{1}{2} K-1 \geqslant c_{4} T \log ^{1 / 8} T, \quad c_{4}>0
$$

Теорема доказана.

ЗАмЕчАнИЕ. Идея сравнивать величины $j_{1}^{\alpha}(t)$ и $j_{2}^{\alpha}(t), 0<\alpha<1$, дала возможность изучать нули линейных комбинаций $L$-функций Дирихле и нули $\zeta(s)$ на очень коротких промежутках критической прямой (см. [7]-[10]).

\section{СПИСОК ЦИТИРОВАННОЙ ЛИТЕРАТУРЫ}

[1] Hardy G. H., Littlewood J. E. The zeros of Riemann's zeta-functions on the critical line // Math. Z. 1921. V. 10. P. 283-317.

[2] Selberg A. On the zeros of Riemann's zeta-function // Skr. Norske Vid. Akad. V. 10. Oslo, 1942.

[3] Ramachandra K. On the mean-value and omega-theorems for the Riemann zeta-function. Bombay: Tata Inst. of Fundam. Research, 1995.

[4] Heath-Brown D. R. Fractional moments of the Riemann zeta-function // J. London Math. Soc. 1981. V. 24. P. 65-78.

[5] Jutila M. On the value distribution of the zeta-function on the critical line // Bull. London Math. Soc. 1983. V. 15. P. 513-518.

[6] Карацуба А. А. Дзета-функция Римана и ее нули // УМН. 1985. Т. 40. № 5 (245). С. 19-70.

[7] Карацуба А. А. О нулях функции Дэвенкорта-Хейльбронна, лежащих на критической прямой // Изв. АН СССР. Сер. матем. 1990. Т. 54. № 2. С. 303-315.

[8] Карацуба А.А. О нулях специального вида функций, связанных с рядами Дирихле // Изв. АН СССР. Сер. матем. 1991. Т. 55. № 3. С. 483-514.

[9] Карацуба А. А. О количестве нулей дзета-функции Римана, лежащих на почти всех коротких промежутках критической прямой // Изв. АН СССР. Сер. матем. 1992. Т. 56. № 2. C. $372-397$.

[10] Карацуба А. А. Новый подход к проблеме нулей некоторых рядов Дирихле // Тр. МИАН. 1994. T. 207. C. $180-196$. 